\title{
The association of serum soluble Klotho levels and residual diuresis and overhydration in peritoneal dialysis patients
}

\author{
Dorota Sikorska, ${ }^{1, A-F}$, Krzysztof Pawlaczyk2,A,F, Ewa Baum ${ }^{3, E, F}$, Maria Wanic-Kossowska ${ }^{2, B, F}$, Natasza Czepulis, ${ }^{4, B, F}$, \\ Joanna Łuczak ${ }^{2, B, F}$, Włodzimierz Samborski ${ }^{1, E, F}$, Andrzej Oko ${ }^{2, A, E, F}$ \\ 1 Department of Rheumatology and Rehabilitation, Poznan University of Medical Sciences, Poland \\ ${ }^{2}$ Department of Nephrology, Transplantology and Internal Medicine, Poznan University of Medical Sciences, Poland \\ ${ }^{3}$ Department of Philosophy and Bioethics, Poznan University of Medical Sciences, Poland \\ ${ }^{4}$ Department of Pathophysiology, Poznan University of Medical Sciences, Poland \\ A - research concept and design; $\mathrm{B}$ - collection and/or assembly of data; $\mathrm{C}$ - data analysis and interpretation; \\ $D$ - writing the article; $E$ - critical revision of the article; $F$ - final approval of the article
}

Address for correspondence

Dorota Sikorska

E-mail: dorotasikorska@ump.edu.pl

Funding sources

The study was supported with a Poznan University of Medical Sciences Grant for Young Researchers (No. 502-14-02225363-99663).

Conflict of interest

None declared

Acknowledgements

We would like to thank Janusz Witowski for comments that greatly improved the manuscript.

Received on February 25, 2018

Reviewed on May 21, 2018

Accepted on February 18, 2019

Published online on March 11, 2019

Cite as

Sikorska D, Pawlaczyk K, Baum E, et al. The association of serum soluble Klotho levels and residual diuresis and overhydration in peritoneal dialysis patients. Adv Clin Exp Med. 2019;28(10):1345-1349. doi:10.17219/acem/104552

DOI

10.17219/acem/104552

Copyright

Copyright by Author(s)

This is an article distributed under the terms of the

Creative Commons Attribution Non-Commercial License

(http://creativecommons.org/licenses/by-nc-nd/4.0/)

\section{Abstract}

Background. Klotho, originally identified as an anti-aging factor, is a transmembrane protein expressed in the kidney. It has been reported that Klotho deficiency could be associated with a loss of residual renal function and cardiovascular complications in peritoneal dialysis (PD) patients.

Objectives. The main aim of the study was to evaluate whether serum levels of Klotho correlate with residual diuresis and hydration status in PD patients.

Material and methods. The cross-sectional study involved 57 PD patients $\geq 18$ years of age who had been on PD $\geq 3$ months. Serum Klotho was measured using high-sensitivity enzyme-linked immunosorbent assay (ELISA). Hydration status was assessed with bioimpedance analysis (BIA).

Results. Serum levels of soluble Klotho ranged from $100 \mathrm{pg} / \mathrm{mL}$ to $700 \mathrm{pg} / \mathrm{mL}$. The patients were divided into 2 subgroups, with Klotho levels below and above the median ( $260 \mathrm{pg} / \mathrm{mL})$. The data revealed a tendency for lower residual diuresis $(1.3 \pm 1.0 \mathrm{~L}$ vs $1.8 \pm 0.8 \mathrm{~L} ; \mathrm{p}=0.055)$ in patients with lower levels of Klotho in serum. Serum Klotho correlated negatively with overhydration according to $B I A(r=-0.27 ; p=0.044)$ and positively with residual diuresis $(r=0.26 ; p=0.045)$.

Conclusions. Soluble Klotho correlates inversely with hydration status in BIA. Residual urine output, but not dialysis parameters, could be associated with the levels of serum soluble Klotho in PD patients.

Key words: peritoneal dialysis, Klotho, overhydration 
Klotho was first identified in mice as an anti-aging factor. ${ }^{1}$ Full-length alpha-Klotho is a single-pass transmembrane protein that exists in 2 forms, membrane and secreted Klotho, which have different functions. ${ }^{2}$ The membrane form acts as a co-receptor for fibroblast growth factor-23 (FGF-23) and plays an important role in calcium-phosphate metabolism. ${ }^{2,3}$ Soluble Klotho (called $\alpha$-Klotho, with molecular mass $130-\mathrm{kDa}$ ) seems to function as a humoral factor with various biological effects, and it works independently of FGF-23 signaling. ${ }^{4}$ Among the pleiotropic actions that soluble Klotho is responsible for are tissue protection from oxidative stress, fibrosis and apoptotic stimuli; regulation of blood phosphate and vitamin D3 levels; and the activity of multiple cell surface calcium and potassium ion channels. ${ }^{2,3,5}$ There are data suggesting that secreted Klotho exerts phosphaturic effects independently of FGF-23. ${ }^{3}$ Soluble Klotho has also been associated with protective effects against vascular calcification. ${ }^{6}$ Furthermore, soluble Klotho could be a potential biomarker for predicting adverse renal outcomes in patients with advanced chronic kidney disease (CKD). ${ }^{7-9}$ However, the exact diagnostic and therapeutic role of Klotho in humans is not fully known yet. ${ }^{3}$

Klotho is expressed in several organs, including the parathyroid glands, the choroid plexus of the brain and, predominantly, in the distal tubular epithelial cells of the kidney. ${ }^{10}$ The kidney is the major source of Klotho in humans, and this organ is involved in Klotho homeostasis, responsible for producing and releasing Klotho into the circulation. ${ }^{11}$ Patients with CKD display decreased Klotho gene expression in several tissues, including the kidney. This results in reduced levels of circulating Klotho. Serum soluble Klotho is decreased in all stages of CKD, especially in dialysis patients. ${ }^{8,11}$ It is likely that serum soluble Klotho protein concentration is related to residual renal function. ${ }^{8}$ However, the relationship between the soluble form of Klotho and residual renal function in chronic peritoneal dialysis (PD) patients remains poorly understood.,

The aim of this study was to assess whether serum soluble Klotho levels could be associated with residual renal function and hydration status in PD patients. To the best of our knowledge, this is the first study evaluating the relationship between serum Klotho and hydration status as assessed with bioimpedance analysis (BIA) in patients on PD.

\section{Material and methods}

\section{Patients}

This investigator-initiated cross-sectional study involved 57 Caucasian patients undergoing PD in 3 regional dialysis centers. The inclusion criteria were: age $\geq 18$ years, time on $\mathrm{PD} \geq 3$ months, and informed consent. The exclusion criteria were: any acute inflammatory disease within 12 weeks prior to enrolment, amputated limbs and cardiac pacemakers or implantable cardioverter defibrillators. The study was approved by the Poznan University of Medical Sciences Bioethics Committee (No. 424/13) and informed consent was obtained from all the participants included in the study.

\section{Volume status}

A peritoneal equilibration test (PET) using a 4-hour dwell of $2.27 \%$-glucose dialysate was used to assess peritoneal membrane transport. ${ }^{12}$

Hydration status was assessed with bioimpedance spectroscopy using the Body Composition Monitor (BCM) (Fresenius Medical Care GmbH, St. Wendel, Germany). ${ }^{13}$ The measurements were performed in the supine position under standardized conditions. Values below $-1.1 \mathrm{~L}$ and above $+1.1 \mathrm{~L}$ corresponded to hypovolemia and hypervolemia, respectively. ${ }^{13}$ Clinical assessments of hydration status were based on blood pressure measurements and on the symptoms of overhydration: the presence of dyspnea, peripheral edema and jugular vein distension.

\section{Laboratory tests}

Samples of serum were collected at the time of the clinical examinations in a fasting state. Serum was aliquoted and stored at $-80^{\circ} \mathrm{C}$ until assayed in batches. Serum Klotho was measured using the Human Soluble $\alpha$-Klotho Assay Kit (Immuno-Biological Laboratories Co. Ltd., Fujioka, Japan) with a sensitivity of $6.15 \mathrm{pg} / \mathrm{mL}$. The immunoassays were performed according to the manufacturer's instructions. All other laboratory tests were performed in the hospital central laboratory using routine methods.

\section{Statistical methods}

Statistical analyses were performed using STATISTICA v. 10.0 software (StatSoft Polska, Kraków, Poland). The normality of the data distribution was checked with the Shapiro-Wilk test. The data is presented as medians and interquartile ranges or percentages, as appropriate. Differences between unpaired data were analyzed with the Mann-Whitney U test. Categorical data was analyzed with the $\chi^{2}$ test. Correlations between variables were analyzed with Spearman's rank correlation coefficient. Differences were considered significant at $\mathrm{p}<0.05$.

\section{Results}

The study analyzed 57 consecutive PD patients. Serum levels of soluble Klotho ranged from 100 to 700 pg/mL. The patients were divided into 2 subgroups: those with Klotho levels below the median $(260 \mathrm{pg} / \mathrm{mL})$ and those with levels above the median. We decided on such subdivision of patients because there are no clear ranges of reference values for Klotho. ${ }^{4}$ The patients' characteristics in relation to their serum Klotho levels are shown in Table 1. 
Clinical features of overhydration were observed in 19\% of the patients (11/57). However, BIA found excessive hydration retention in as many as $44 \%$ of the patients (25/57). In addition, there was a significant difference in the distribution of those patients between the Klotho subgroups (Table 1). Surprisingly, there were no statistically significant differences in Klotho levels between patients with overhydration according to BIA and those without (250 (216-327) pg/mL vs 290 (223-399) pg/mL; p = 0.172).

The data revealed a tendency toward higher fluid overload in BIA in patients with lower levels of Klotho in serum
(Table 1), although these were not statistically significant differences. Also, patients with lower serum Klotho had significantly lower hematocrit and hemoglobin levels. These patients also tended to have lower residual diuresis. However, there was no relationship between serum Klotho levels and dialysis parameters (Table 1).

As expected, serum Klotho correlated negatively with overhydration according to BIA $(r=-0.27 ; \mathrm{p}=0.044)$ (Fig. 1). Moreover, there was a positive correlation between Klotho and residual diuresis $(\mathrm{r}=0.26$; $\mathrm{p}=0.045)$ (Fig. 2).

Table 1. Patients' characteristics in relation to serum Klotho levels. Data are presented as medians (interquartile ranges) or as percentages

\begin{tabular}{|c|c|c|c|}
\hline Variables & $\begin{array}{l}\text { Lower levels of Klotho }(<260 \mathrm{pg} / \mathrm{mL}) \\
\qquad(\mathrm{n}=28)\end{array}$ & $\begin{array}{l}\text { Higher levels of Klotho ( } \geq 260 \mathrm{pg} / \mathrm{mL}) \\
\qquad(\mathrm{n}=29)\end{array}$ & $\begin{array}{l}\text { Mann-Whitney } \\
\text { or } X^{2} \text { test }\end{array}$ \\
\hline \multicolumn{4}{|c|}{ Demographic and PD-related parameters } \\
\hline Men [\%] & $14(50)$ & $14(48)$ & 0.896 \\
\hline Age [years] & $60(42-70)$ & $55(39-67)$ & 0.375 \\
\hline BMI $\left[\mathrm{kg} / \mathrm{m}^{2}\right]$ & $24.4(21.0-30.3)$ & $26.0(23.9-29.1)$ & 0.664 \\
\hline Diabetic nephropathy, n [\%] & $8(29)$ & $7(24)$ & 0.704 \\
\hline $\mathrm{DM}[\%]$ & $11(39)$ & $9(31)$ & 0.514 \\
\hline Time on PD [months] & $25(15-38)$ & $33(16-68)$ & 0.123 \\
\hline APD mode, $n[\%]$ & $10(36)$ & $6(21)$ & 0.207 \\
\hline Ultrafiltration [mL/day] & $1,200(500-2,200)$ & $1,000(900-1,500)$ & 0.766 \\
\hline Residual diuresis [mL/day] & $1,300(500-2,200)$ & $1,650(1,100-2,400)$ & 0.054 \\
\hline Residual diuresis < 500 mL/day [\%] & $7(25)$ & $1(3)$ & 0.019 \\
\hline Solute removal [Kt/V] & $2.3(2.1-3.5)$ & $2.8(2.3-3.3)$ & 0.421 \\
\hline Creatinine clearance [L/week] & $92.8(75.4-120.2)$ & $99.4(68.8-136.1)$ & 0.799 \\
\hline 4-h D/P creatinine in PET & $0.68(0.58-0.72)$ & $0.63(0.55-0.71)$ & 0.402 \\
\hline Transport status, n (\% H/HA) & $16(57)$ & $12(41)$ & 0.234 \\
\hline \multicolumn{4}{|c|}{ Blood tests and biochemical parameters } \\
\hline Hematocrit [\%] & $33.1(31.5-37.4)$ & $37.1(33.4-38.5)$ & 0.018 \\
\hline Hemoglobin [g/dL] & $11.1(10.5-12.4)$ & $12.5(11.3-13.4)$ & 0.016 \\
\hline $\mathrm{CRP}[\mathrm{mg} / \mathrm{L}]$ & $5.6(2.6-8.4)$ & $2.7(1.3-8.2)$ & 0.123 \\
\hline Albumin $[\mathrm{g} / \mathrm{dL}]$ & $3.8(3.5-4.1)$ & $4.0(3.7-4.2)$ & 0.107 \\
\hline Total cholesterol [mg/dL] & $184(167-201)$ & $198(169-216)$ & 0.263 \\
\hline Calcium [mmol/L] & $9.1(8.5-9.5)$ & $9.0(8.7-9.3)$ & 0.936 \\
\hline Phosphorus [mmol/L] & $5.3(3.9-6.2)$ & $5.1(4.3-6.1)$ & 0.707 \\
\hline PTH $[\mathrm{pg} / \mathrm{mL}]$ & $278(178-401)$ & $340(299-490)$ & 0.080 \\
\hline FGF-23 [pg/mL] & $8.9(3.2-41.6)$ & $18.3(5.6-44.4)$ & 0.457 \\
\hline \multicolumn{4}{|c|}{ Hydration status } \\
\hline $\mathrm{OH}$ in $\mathrm{BIA}[\mathrm{L}]$ & $2.0(0.6-3.0)$ & $0.8(0.2-1.6)$ & 0.066 \\
\hline $\mathrm{OH}$ in $\mathrm{BIA}[\%]$ & $2.3(0.9-3.9)$ & $1.0(0.3-2.5)$ & 0.056 \\
\hline Overhydration in BIA > $1.1 \mathrm{~L}$ [\%] & $16(57)$ & $9(31)$ & 0.047 \\
\hline Pts with edema, n [\%] & $7(25)$ & $4(14)$ & 0.284 \\
\hline $\mathrm{SBP}[\mathrm{mm} \mathrm{Hg}]$ & $130(120-150)$ & $130(120-145)$ & 0.705 \\
\hline $\mathrm{DBP}[\mathrm{mm} \mathrm{Hg}]$ & $80(70-95)$ & $80(70-90)$ & 0.497 \\
\hline Number of antihypertensives & $3(2-4)$ & $4(2-4)$ & 0.215 \\
\hline
\end{tabular}

BMI - body mass index; DM - diabetes mellitus; PD - peritoneal dialysis; APD - automated peritoneal dialysis; $\mathrm{H}$ - high; HA - high-average; CRP - C-reactive protein; PTH - parathyroid hormone; FGF-23 - fibroblast growth factor 23; OH - overhydration; BIA - bioimpedance analysis; SBP - systolic blood pressure; DBP - diastolic blood pressure. 


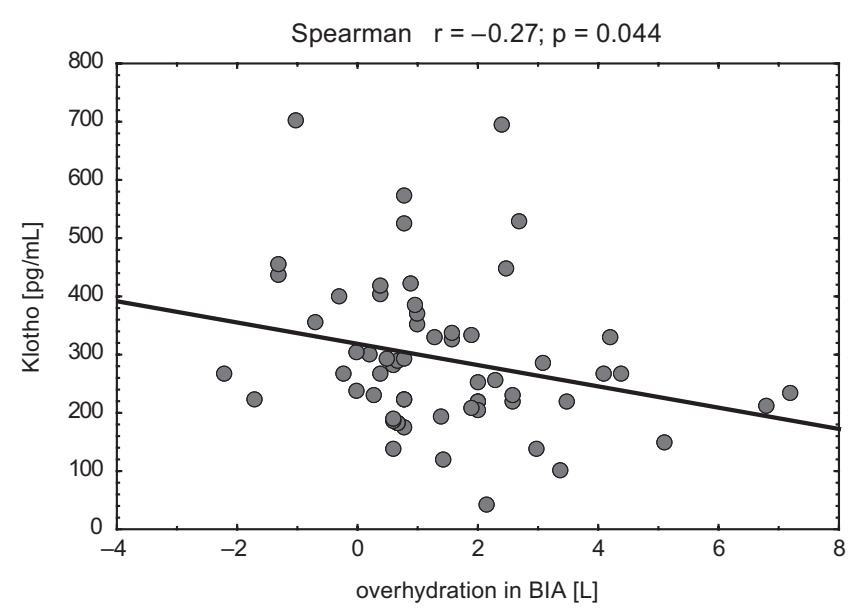

Fig. 1. Correlation between hydration status in bioimpedance analysis (BIA) and serum Klotho

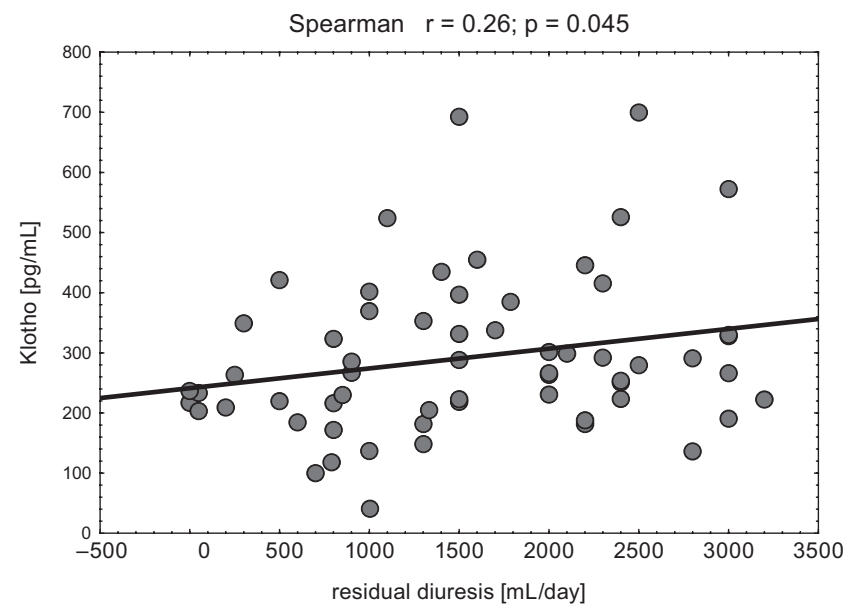

Fig. 2. Correlation between residual diuresis and serum Klotho

\section{Discussion}

The most important observation in our study was that serum soluble Klotho levels correlate inversely with hydration status in BIA. The results of the study suggest that residual urine output, but not dialysis parameters, could be associated with the levels of serum Klotho in PD patients.

Earlier studies indicated that serum soluble Klotho levels are positively associated with renal function and are significantly decreased in the later stages of CKD. ${ }^{14,15}$ At the same time, Klotho concentrations are probably independent of PD parameters themselves. ${ }^{16}$ However, the relationship between serum Klotho and residual renal function is not so obvious in PD patients. ${ }^{79}$ A previous study revealed that the total amount of urinary excreted Klotho, but not the serum level of soluble Klotho, may be a potential biomarker for assessing the residual renal function among PD patients. ${ }^{9}$ In 2012, Golembiewska et al. demonstrated that serum soluble Klotho concentrations were negatively correlated with a 24-hour diuresis, but not with residual renal function. ${ }^{7}$

There has been a strong focus on residual renal function as a significant survival predictor for dialysis patients, ${ }^{17,18}$ but the precise mechanism by which residual diuresis is linked to morbidity and mortality among dialysis patients has yet to be determined. ${ }^{19}$ The presence of residual renal function is associated with better preservation of the renal endocrine and metabolic functions, and facilitates the maintenance of good hydration status. ${ }^{20,21}$ It is likely that residual urinary volume is the main predictor of overhydration in patients on PD. ${ }^{22}$ Moreover, our study demonstrated a significant correlation between residual urine output and hydration status $(\mathrm{r}=-0.36 ; \mathrm{p}=0.004)$.

It is possible that the relationship between residual diuresis and soluble Klotho in our study results from hydration status. However, to rule out the effect of dilution of serum Klotho, albumin concentrations were also assessed, and there was no relationship between Klotho levels and albumin concentrations. At the same time, we did not detect any correlation between overhydration in BIA and blood pressure, which should be influenced by an increase in plasma volume. This may suggest that overhydration is related to interstitial fluid retention rather than hypervolemia. ${ }^{23}$ It is possible that overhydration per se affects soluble Klotho levels.

The introduction of BIA has made it possible to assess hydration status in PD patients more accurately. ${ }^{13}$ Recent studies have revealed that BIA-evaluated overhydration is common in dialysis patients, and is associated with loss of residual renal function and inflammation. ${ }^{24,25}$ Increasing evidence suggests that overhydration predicts all-cause mortality. ${ }^{26}$ However, the relationship between overhydration and Klotho is not yet known. To the best of our knowledge, this is the first study evaluating the relationship between serum Klotho and hydration status in BIA in patients on PD.

Although this study provides new information on the relationship between residual diuresis, hydration status and soluble Klotho among PD subjects, the results should be interpreted within the context of the study limitations. The patient population was relatively small, which means that the study may be statistically underpowered. An additional limitation is that we cannot provide data on urinary and dialysate Klotho excretion. Further research is therefore needed.

\section{Conclusions}

Residual urine output, but not dialysis parameters, could be associated with the levels of serum soluble Klotho in PD patients. Soluble Klotho correlates inversely with hydration status in BIA. 


\section{References}

1. Kuro-o M, Matsumura Y, Aizawa H, et al. Mutation of the mouse klotho gene leads to a syndrome resembling ageing. Nature. 1997;390(6655): 45-51.

2. Olauson $\mathrm{H}$, Mencke R, Hillebrands JL, Larsson TE. Tissue expression and source of circulating alphaKlotho. Bone. 2017;100:19-35.

3. Golembiewska E, Stepniewska J, Kabat-Koperska J, Kedzierska K, Domanski M, Ciechanowski K. The role of Klotho protein in chronic kidney disease: Studies in animals and humans. Curr Protein Pept Sci. 2016;17(8):821-826.

4. Wang Y, Sun Z. Current understanding of klotho. Ageing Res Rev. 2009;8(1):43-51.

5. Xu Y, Sun Z. Molecular basis of Klotho: From gene to function in aging Endocr Rev. 2015;36(2):174-193.

6. Lim K, Lu TS, Molostvov G, et al. Vascular Klotho deficiency potentiates the development of human artery calcification and mediates resistance to fibroblast growth factor 23. Circulation. 2012;125(18): 2243-2255.

7. Golembiewska E, Safranow K, Kabat-Koperska J, Myslak M, Ciechanowski K. Serum soluble Klotho protein level is associated with residual diuresis in incident peritoneal dialysis patients. Acta Biochim Pol. 2013;60(2):191-194.

8. Liu QF, Ye JM, Yu LX, et al. Plasma s-Klotho is related to kidney function and predicts adverse renal outcomes in patients with advanced chronic kidney disease. J Investig Med. 2017;66(3):669-675.

9. Akimoto T, Shiizaki K, Sugase $T$, et al. The relationship between the soluble Klotho protein and the residual renal function among peritoneal dialysis patients. Clin Exp Nephrol. 2012;16(3):442-447.

10. Gigante M, Lucarelli G, Divella C, et al. Soluble serum alphaKlotho is a potential predictive marker of disease progression in clear cell renal cell carcinoma. Medicine (Baltimore). 2015;94(45):e1917.

11. Hu MC, Shi M, Zhang J, et al. Renal production, uptake, and handling of circulating alphaKlotho. J Am Soc Nephrol. 2016;27(1):79-90.

12. Twardowski ZJ, Nolph KD, Khanna R, et al. Peritoneal equilibration test. Perit Dial Int. 1987;7(3):138-148.

13. Davies SJ, Davenport A. The role of bioimpedance and biomarkers in helping to aid clinical decision-making of volume assessments in dialysis patients. Kidney Int. 2014;86(3):489-496.
14. Shimamura Y, Hamada K, Inoue K, et al. Serum levels of soluble secreted alpha-Klotho are decreased in the early stages of chronic kidney disease, making it a probable novel biomarker for early diagnosis. Clin Exp Nephrol. 2012;16(5):722-729.

15. Seibert E, Radler D, Ulrich C, Hanika S, Fiedler R, Girndt M. Serum klotho levels in acute kidney injury. Clin Nephrol. 2017;87 (2017)(4): 173-179.

16. Oh HJ, Nam BY, Lee MJ, et al. Decreased circulating klotho levels in patients undergoing dialysis and relationship to oxidative stress and inflammation. Perit Dial Int. 2015;35(1):43-51.

17. Lee MJ, Park JT, Park KS, et al. Prognostic value of residual urine volume, GFR by 24-hour urine collection, and eGFR in patients receiving dialysis. Clin J Am Soc Nephrol. 2017;12(3):426-434.

18. Diaz-Buxo JA, White SA, Himmele R. The importance of residual renal function in peritoneal dialysis patients. Adv Perit Dial. 2013;29:19-24.

19. Liu X, Dai C. Advances in understanding and management of residual renal function in patients with chronic kidney disease. Kidney Dis (Basel). 2017;2(4):187-196.

20. Sikorska D, Pawlaczyk K, Olewicz-Gawlik A, et al. The importance of residual renal function in peritoneal dialysis. Int Urol Nephrol. 2016; 48(12):2101-2108.

21. Vilar E, Farrington K. Emerging importance of residual renal function in end-stage renal failure. Semin Dial. 2011;24(5):487-494.

22. Jung ES, Sung JY, Han SY, et al. Residual urinary volume is a predictor of overhydration in patients on peritoneal dialysis. Tohoku J Exp Med. 2014;233(4):295-300.

23. Sikorska D, Pawlaczyk K, Roszak M, et al. Preliminary observations on the association between serum IL- 6 and hydration status and cardiovascular risk in patients treated with peritoneal dialysis. Cytokine. 2016;85:171-176.

24. Van Biesen W, Williams JD, Covic AC, et al. Fluid status in peritoneal dialysis patients: The European Body Composition Monitoring (EuroBCM) study cohort. PLoS.ONE. 2011;6(2):e17148.

25. Fan $\mathrm{S}$, Sayed RH, Davenport A. Extracellular volume expansion in peritoneal dialysis patients. Int J Artif Organs. 2012;35(5):338-345.

26. Paniagua R, Ventura MD, Avila-Diaz M, Hinojosa-Heredia H, MendezDuran A, Cueto-Manzano A, et al. NT-proBNP, fluid volume overload and dialysis modality are independent predictors of mortality in ESRD patients. Nephrol Dial Transplant. 2010;25(2):551-557. 